\title{
Development and Validation of a Method for Determination of Encapsulation Efficiency of CPT-11/DSPE-mPEG2000 Nanoparticles
}

Chan Lï, Xianlei Lï, Shuyi Li, Yuhua Weng, Kaiyuan Wang, Tingbin Zhang, Shizhu Chen, Xuexia Lu, Yonggang Jiang, Jing Xu* and Xingjie Liang*

\#Authors contributed equally

CAS Key Laboratory for Biological Effects of Nanomaterials and Nanosafety, National Center for Nanoscience and Technology, No. 11 Beiyitiao, Zhongguancun, Beijing 100190, China

\begin{abstract}
Herein we report development and validation of the method for evaluating the encapsulation efficiency of a micelle-based nanosystem composed of irinotecan hydrochloride (CPT-11) and an amphiphilic molecule DSPEmPEG2000. The results showed that the centrifugation method can be used for separation of free drug, a critical step in measuring encapsulation efficiency, and the EE of three batches of CPT-11/DSPE-mPEG2000 micelles was $90.0 \% \pm 1.0 \%$. The results also indicated that the conditions used in the process have to be optimized to acquire reliable data.
\end{abstract}

Keywords: Irinotecan hydrochloride; Micelle; Encapsulation efficiency; DSPE-mPEG2000

\section{Introduction}

Nano-sized systems especially nanoparticles (NPs) have been extensively explored for encapsulating anti-cancer drugs of low solubility and facilitating better delivery of drug molecules to tumors through the enhanced permeability and retention (EPR) effect. The introduction of NPs can thus substantially reduce undesired side effects of drug formulations through removal of excipients associated with the side effects and enhance therapeutic efficacy by altering the biodistribution profiles and pharmacokinetics of the free drug [1]. The encapsulation of drug molecules within the "Trojan horse" nanosystems is the key in harnessing the power of nanotechnology for development of better anti-cancer therapeutics [2]. Because of this, the determination of the encapsulation efficiency (EE) which is defined as the percentage of drug molecules successfully entrapped within the NPs is regarded as a key step in characterizing the quality of the nanoformulations $[3,4]$.

Among the various types of NPs currently being investigated, micelle is a relatively simple system formed by the spontaneous self-assembly of block copolymers in aqueous solution [5,6]. The micelle is usually comprised of a hydrophobic core and surrounding hydrophilic corona. The drug molecules can be entrapped within the core of the micelle system through various interactions, mainly hydrophobic-hydrophobic, and be protected from prematured release. For example, Genexol-PM, a micelle form of the paclitaxel composed of polyethyleneglycol (PEG)-polylactide, has shown both increased efficacy and less toxicity and has now been approved for marketing in several countries and currently in late stage clinical trials in the USA. In addition, there are ongoing clinical studies for other micelle-based anticancer drug formulations such as NK911, NK012, NK105 (all three are developed by Nippon Kayaku Co. Ltd.), SP1049C (Supratech Pharma Inc.), and BIND-014 (Bind Therapeutics) [7].

Micelles are formed when the concentration of block copolymers goes above the critical micelle concentration (CMC) [8]. This process is usually driven by the hydrophobic-hydrophobic interaction. Below the CMC, the block copolymers will exist in a freely dispersed state. Above the $\mathrm{CMC}$, the assembled structures and the free molecules are in a state of dynamic equilibrium and the system is thermodynamically stable. Therefore, a critical step in the analysis of EE is to separate untrapped, free drug molecules from the drug incorporated into the micelle.

This paper reports the determination of EE for a micelle system composed of irinotecan hydrochloride (CPT-11) and 1,2-distearoylsn-glycero-3-phosphoethanolamine- $\mathrm{N}$-[methoxy(polyethylene glycol)-2000] (DSPE-mPEG2000). The solution form of CPT-11 is approved to be used alone or with other drugs to treat metastasized colorectal cancer and a nanoliposomal form of irinotecan (developed by Merrimack Pharmaceuticals) has just got approved by US FDA for treatment of metastatic pancreatic cancer [7]. The DSPE-mPEG2000 is an amphiphilic molecule that is regarded as a GRAS (generally regarded as safe) material by FDA and has been widely used in pharmaceutical research [9]. In this paper, we examined three strategies including dialysis method, centrifugation method and a gel chromatography method for separation of untrapped drug molecule from the micellar system $[3,10,11]$. The amount of the drug was characterized using high performance liquid chromatography (HPLC) coupled with UVVis detector or UV spectrometry. Herein we want to highlight the importance of method validation during the analysis of EE. The results indicated that among the three methods investigated, the centrifugation method is the most appropriate one for separation of free drug for this micelle system and the EE of three batches of CPT-11/DSPE-PEG2000 micelles was determined to be $90.0 \% \pm 1.0 \%$.

\section{Methods}

\section{Synthesis of CPT-11/DSPE-mPEG2000 micelle}

Irinotecan hydrochloride-encapsulated DSPE-mPEG2000 micelles were pared by a green method. Briefly, $166.7 \mathrm{mg}$ of DSPE-mPEG2000 was dissolved in $1 \mathrm{~mL}$ of lactose aqueous solution (5 wt.\%) under stirring to form a clear and colorless system. Then $40 \mathrm{mg}$ of irinotecan

*Corresponding authors: Jing Xu, National Center for Nanoscience and Technology, No. 11 Beiyitiao, Zhongguancun, Beijing 100190, China, Tel: +8601082545530; E-mail: xujing@nanoctr.cn

Xingjie Liang, National Center for Nanoscience and Technology, No. 11 Beiyitiao, Zhongguancun, Beijing 100190, China, Tel: +8601082545530; E-mail: liangxi@nanoctr.cn

\section{Received May 05, 2016; Accepted May 23, 2016; Published May 30, 2016}

Citation: Li C, Li X, Li S, Weng Y, Wang K, et al. (2016) Development and Validation of a Method for Determination of Encapsulation Efficiency of CPT11/DSPE-mPEG2000 Nanoparticles. Med chem (Los Angeles) 6: 345-348. doi:10.4172/2161-0444.1000368

Copyright: ( $2016 \mathrm{Li} \mathrm{C}$, et al. This is an open-access article distributed under the terms of the Creative Commons Attribution License, which permits unrestricted use, distribution, and reproduction in any medium, provided the original author and source are credited. 
hydrochloride trihydrate was added into the DSPE-mPEG2000/ lactose $/ \mathrm{H}_{2} \mathrm{O}$ system under stirring to give a transparent and light yellow colloidal solution, which was confirmed by its obvious Tyndall effect. The solution was then filtered using a $0.22 \mu \mathrm{m}$ membrane and lyophilized to obtain yellowish powder.

\section{Characterization of CPT-11/DSPE-mPEG2000 micelle}

The micelle solution was prepared by dissolving the CPT-11/DSPEmPEG2000 powder in medium. The CPT-11/DSPE-mPEG2000 micelles were imaged by a transmission electron microscope (FEI Tecnai $G^{2} 20$ S-TWIN) and the hydrodynamic diameter was measured by dynamic light scattering (Malvern Instruments Inc., Zetasizer Nano ZS). For zeta potential measurements, the particles were dispersed in $1 \mathrm{mM}$ potassium chloride at a concentration of $10 \mu \mathrm{g} / \mathrm{mL}$ and tested by a Zetasizer Nano Analyzer (Malvern Instruments Inc., Zetasizer Nano ZS).

\section{Separation of free CPT-11 for determination of EE}

A drug micelle solution of CPT-11 was prepared by dissolving the lyophilized CPT-11/DSPE-mPEG2000 powder in medium.

The gel filtration chromatography method: $6 \mathrm{~mL}$ of wet Sephadex G-50 (from pharmacia) material was packed into the column $(1 \mathrm{~cm}$ $\times 10 \mathrm{~cm})$ as instructed and $200 \mu \mathrm{L}$ of the CPT-11/DSPE-mPEG2000 drug solution $(2.8 \mathrm{mg} / \mathrm{mL})$ was carefully loaded into the Sephadex G-50 material from the top of the column. Eluting solution $(200 \mathrm{~mL})$ was then added and the solution was eluted out at a speed of $0.7 \mathrm{~mL} / \mathrm{min}$. A UV-vis detector (MC-UV200, Company of mancang technology, Beijing) was used to monitor the absorption of the eluted solution real-timely at the wavelength of $254 \mathrm{~nm}$. The eluted solution was then collected and HPLC-UV was employed to determine the concentration.

The dialysis method: $2 \mathrm{~mL}$ CPT-11/DSPE-mPEG2000 particle solution was placed in dialysis bag (regenerated cellulose, MW 3500) and dialyzed against $1000 \mathrm{~mL}$ of medium. After 24 hours, the amount of drug that was recovered from the dialysis bag (W (recovered)) was determined by HPLC-UV-vis. The EE was calculated using the equation below:

$$
E E(\%)=\frac{\mathrm{W}(\text { recovered })}{\mathrm{W}(\text { total })} \times 100 \%
$$

Where $\mathrm{W}$ (total) is the total amount of drug in the dialysis bag prior to the procedure, $\mathrm{W}$ (recovered) is the amount of drug that was recovered from the dialysis bag at the end.

Centrifugation method: Centrifugal filters (Merck Millipore, 4 $\mathrm{mL}$, MWCO $30 \mathrm{~K}$ ) were used to separate trapped CPT-11 from free CPT-11. The CPT-11/DSPE-mPEG2000 particle solution was placed in the filter and the centrifugation was done at a speed of $7200 \mathrm{~g} \mathrm{(8321}$ $\mathrm{rpm}$ ), at $4^{\circ} \mathrm{C}$ using a centrifuge manufactured by Beckman Coulter (Allegra 64R). The solution at the bottom of the filter was carefully removed from the device and the concentration of CPT-11 in free form $\left(\mathrm{C}_{\text {free }}\right)$ was determined. The EE was calculated using the equation below:

$$
E E(\%)=\frac{\mathrm{C}(\text { total })-\mathrm{C}(\text { free })}{\mathrm{C}(\text { total })} \times 100 \%
$$

Where C (Total) is the total drug concentration before filtration containing both trapped and free CPT-11, C (free) is the drug concentration in the filtered solution.

\section{Quantification of drug CPT-11 using HPLC-UV method}

The amount of CPT-11 was measured using HPLC (Shimadzu CTO$20 \mathrm{~A})$ with a $\mathrm{C} 18$ rapid resolution column $(4.6 \times 250 \mathrm{~mm}, 5 \mathrm{micron})$. A mobile phase of acetonitrile:methanol:solution $\mathrm{A}=17: 24: 59$ with a flow rate of $1.5 \mathrm{~mL} / \mathrm{min}$ was employed with a column temperature of $40^{\circ} \mathrm{C}$ and a detection wavelength of $254 \mathrm{~nm}$ on the UV-Vis detector (Solution $\mathrm{A}=2.8 \mathrm{~g} / \mathrm{L}$ of $\mathrm{NaH}_{2} \mathrm{PO}_{4} \cdot 2 \mathrm{H}_{2} \mathrm{O}$ and $1.8 \mathrm{~g} / \mathrm{L}$ of 1 -octanesulfonic acid sodium salt in water). The CPT-11 peak appeared at 15.3 minutes and the peak area was compared to the CPT- 11 standard curve to determine the concentration of CPT-11 in the solution. For determination of total amount of CPT-11 in the micelle solution or free CPT-11 solution, 20 $\mu \mathrm{L}$ of the drug solution was injected into the HPLC.

\section{Results and Discussion}

\section{Preparation and characterization of CPT-11/DSPE- mPEG2000 nanoparticle}

The CPT-11/DSPE-mPEG2000 micelles were prepared using a direct solution processing strategy. This "green" synthesis method does not involve any organic solvent and enables the scale-up of nanoparticle production to levels practical for clinical applications. The particles showed a monodispersed size of $15.1 \pm 0.8 \mathrm{~nm}$ based on DLS. Due to the anionic nature of polyethylene glycol, the particles are negatively charged $(\zeta$ potential $=-4.6 \pm 1.3 \mathrm{mV})$. The TEM images were shown in Figure 1).

\section{The gel filtration chromatography method}

The gel filtration chromatography, also referred to as size exclusion chromatography, employs porous gel particles suspended in aqueous medium and has been widely used to separate molecules based on their molecular weight. In the elution process, smaller molecules can enter the pores and have to travel longer distances when move through the column. On the other hand, bigger structures cannot defuse freely and therefore elute earlier. Gel filtration chromatography has been used to remove unencapsulated molecules from nanoparticles especially liposomes [12].

Free CPT-11 solution was loaded on the column and the recovery rate was determined. HPLC was used to measure the amount of CPT11 in the fractions collected. The results indicated that by using $5 \%$ glucose as eluting solution only $10 \%$ of drug was recovered from the gel column and a sample recovery of $98.4 \%$ was achieved in the case of PBS. We further evaluated PBS as the eluting buffer. We built a simple

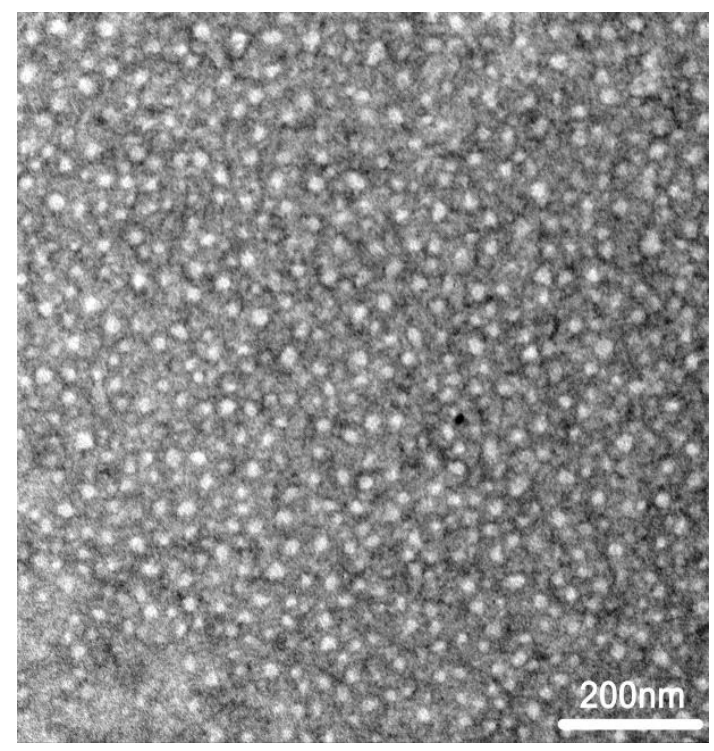

Figure 1: TEM image of the CPT-11/DSPE-mPEG2000 micelles. 
system with a UV-detector at the bottom of the column to acquire realtime elution profiles. Ideally, we expect that two peaks, the first one for the CPT-11 within micelles and the second one for free CPT-11, will appear. The results (Figure 2) showed that by using phosphate buffer solution (PBS), only one peak appeared around $9 \mathrm{~min}$. In this case, the free CPT-11 and trapped CPT-11 were not successfully separated through the elution. It is highly possible that during the elution process, the concentration of the micelles fell below its CMC and the drug was released upon destruction of the micelles. This method is therefore not appropriate for separation of free drug for this system.

\section{The dialysis method}

Dialysis is another method frequently used to isolate nanoparticles from nanoparticle and free drugs mixture. The nanoparticles can be retained within the dialysis bag while the free drug defuses out. In this experiment, we used dialysis bags made from regenerated cellulose with a MWCO of $30 \mathrm{~K}$. However, the recovery results showed that only $20 \%$ of drug can be recovered from the solution inside and outside of the dialysis bag. Due to the stability of CPT-11 under the experiment condition, we rule out the possibility that the CPT-11 degraded in the system. In addition, HPLC results also showed no extra peak from any degraded products. We deduced that the drug has been detained by the dialysis bag and failed to escape. Therefore, the dialysis method does not fit this nanoparticle formulation.

\section{The centrifugation method}

Centrifugation method was used to separate free molecules from the micelles based on their different ability to cross through the membrane with different sizes of pores upon centrifugation [13]. We first evaluated the recovery of free CPT-11 solution in the procedure. The results (Table 1) showed that with more concentrated drug solution, higher recovery rates can be achieved. This might be attributed to the fact that the membrane on the centrifugation filter can only hold a certain amount of drug and when the original concentration is high enough, this absorbed part became negligible. The data also indicated that the MWCO of the membrane does not significantly affect the recovery rate. For further study, we chose the $30000 \mathrm{MWCO}$ and 0.3 to $1 \mathrm{mg} / \mathrm{mL}$ of CPT-11 concentration. We then evaluated the influence of centrifugation time on recovery. The results (Table 2) showed that

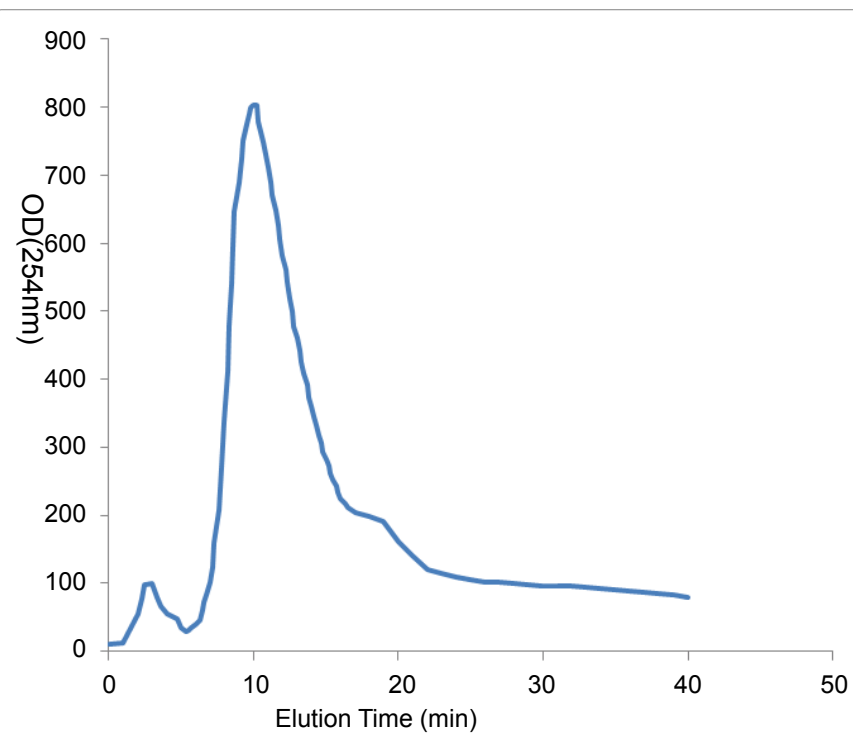

Figure 2: The real-time absorption measured using the gel filtration chromatography as separation method for free CPT-11. centrifugation time has minimal effect on the recovery and $20 \mathrm{~min}$ was chosen so that relatively larger amount of sample can be obtained for analysis. To further confirm that free drug in the presence of micelles can be separated using this strategy, we briefly mixed empty micelles with free drug solution and then performed the above procedure to recover the free drug and the results showed that up to $99 \%$ of free drug could be recovered.

We then used the aforementioned method for analysis of EE of the CPT-11/DSPE-mPEG2000 micelles. The micelle dry powder was suspended in three different media including $0.9 \% \mathrm{NaCl}$ solution, deionized water (DI water) and 5\% glucose at a concentration of $8 \mathrm{mg} /$ $\mathrm{mL}$ of CPT- 11 . The results (Table 3 ) showed that the EE in $0.9 \%$ of $\mathrm{NaCl}$ solution is $76.57 \%$ while the EEs in DI water and $5 \%$ glucose are $95.22 \%$ and $93.32 \%$, respectively. We hypothesized that the encapsulation of CPT-11 within the DSPE-mPEG2000 core might be partially attributed to the electrostatic interaction between the two components and the stability of the micellar structure might be disrupted to some extent due to the ionic strength in $0.9 \% \mathrm{NaCl}$ solution. Based on this result, $5 \%$ glucose was chosen as the dispersion medium for further study.

We then tested the EE of the CPT-11/DSPE-mPEG2000 micelles at different concentrations of CPT-11. The results (Table 4) showed that the EE remained unchanged until the CPT-11 concentration fell below $0.18 \mathrm{mg} / \mathrm{mL}$. However, previous results showed that at low CPT11 concentration, below $0.3 \mathrm{mg} / \mathrm{mL}$, the absorption of the drug on the membrane of centrifugation filter became substantial compared to the free drug solution and therefore cannot be neglected. Because of this, we chose the $2.8 \mathrm{mg} / \mathrm{mL}$ of CPT- 11 as the testing condition due to the fact that the free drug concentration in this solution is around $0.3 \mathrm{mg} /$ $\mathrm{mL}$ and the result is therefore most accurate.

We then used this method to test three batches of CPT-11/DSPEmPEG2000 micelles and results showed an EE of $90.0 \% \pm 0.1 \%$. The solution was incubated at $4^{\circ} \mathrm{C}$ in the darkness and the $\mathrm{EE}$ did not show

\begin{tabular}{|c|c|c|}
\hline Conc. of CPT-11 (mg/mL) & MWCO of membrane & Recovery (\%) \\
\hline \multirow{3}{*}{0.1} & 3000 & $71.0 \%$ \\
\hline & 10000 & $70.9 \%$ \\
\hline & 30000 & $82.7 \%$ \\
\hline \multirow{3}{*}{0.3} & 3000 & $89.3 \%$ \\
\hline & 10000 & $91.4 \%$ \\
\hline & 30000 & $93.7 \%$ \\
\hline \multirow{3}{*}{1} & 3000 & $92.6 \%$ \\
\hline & 10000 & $95.2 \%$ \\
\hline & 30000 & $96.2 \%$ \\
\hline
\end{tabular}

Table 1: Recovery rate from centrifugation filtration.

\begin{tabular}{|c|c|c|}
\hline Conc. of CPT-11 $\mathbf{( m g / m L )}$ & Time (min) & Recovery (\%) \\
\hline \multirow{3}{*}{0.3} & 10 & $93.8 \%$ \\
\cline { 2 - 3 } & 20 & $93.7 \%$ \\
\cline { 2 - 3 } & 30 & $94.3 \%$ \\
\hline \multirow{2}{*}{1} & 10 & $92.3 \%$ \\
\hline \multirow{2}{*}{1} & 20 & $96.3 \%$ \\
\hline
\end{tabular}

Table 2: Recovery rate from centrifugation filtration.

\begin{tabular}{|c|c|}
\hline Medium & EE \\
\hline $0.9 \% \mathrm{NaCl}$ solution & $76.6 \%$ \\
\hline DI water & $95.2 \%$ \\
\hline $5 \%$ glucose & $93.3 \%$ \\
\hline
\end{tabular}

Table 3: EE of the CPT-11/DSPE-mPEG2000 micelles in different medium. 
Citation: Li C, Li X, Li S, Weng Y, Wang K, et al. (2016) Development and Validation of a Method for Determination of Encapsulation Efficiency of CPT-11/DSPE-mPEG2000 Nanoparticles. Med chem (Los Angeles) 6: 345-348. doi:10.4172/2161-0444.1000368

\begin{tabular}{|c|c|}
\hline Concentration Of CPT-11 $\mathbf{( m g} / \mathbf{m L})$ & EE \\
\hline 8 & $93.3 \%$ \\
\hline 2.8 & $89.5 \%$ \\
\hline 1 & $87.5 \%$ \\
\hline 0.8 & $87.8 \%$ \\
\hline 0.6 & $86.6 \%$ \\
\hline 0.4 & $85.8 \%$ \\
\hline 0.2 & $83.0 \%$ \\
\hline 0.18 & $83.2 \%$ \\
\hline 0.12 & $78.5 \%$ \\
\hline
\end{tabular}

Table 4: EE of the CPT-11/DSPE-mPEG2000 micelles at different CPT-11 concentration.

\begin{tabular}{|c|c|}
\hline Time & $\mathbf{2 . 8} \mathbf{~ m g} / \mathbf{m L}$ \\
\hline $0 \mathrm{~h}$ & $89.5 \%$ \\
\hline $9 \mathrm{~h}$ & $89.3 \%$ \\
\hline $24 \mathrm{~h}$ & $89.6 \%$ \\
\hline
\end{tabular}

The CPT-11/DSPE-mPEG2000 micelle solution was incubated in the darkness at $4^{\circ} \mathrm{C}$

Table 5: Stability of the CPT-11/DSPE-mPEG2000 micelles after incubation.

any change, indicating that the micelle system is relatively stable when suspended in the medium (Table 5). We also incubated the dry powder of CPT-11/DSPE-mPEG2000 micelles at $60^{\circ} \mathrm{C}$ and the results also showed that EE remained the same over 6 months.

\section{Conclusion}

We examined three methods for evaluating the EE of a CPT-11/ DSPE-mPEG2000 micellar system. Through validation of methods and conditions, an ultracentrifugation method was established to measure the EE of the CPT-11/DSPE-mPEG2000 micelles. Our results showed that an appropriate condition has to be used to achieve more accurate and solid data when evaluating encapsulation efficiency of nanoparticlebased drug formulations.

\section{Funding}

This work is supported by the "Strategic Priority Research Program" of the Chinese Academy of Sciences Grant No. XDA09030301.

\section{References}

1. Chow EK, Ho D (2013) Cancer nanomedicine: from drug delivery to imaging Sci Transl Med 5: $216 r v 4$.

2. Zamboni WC, Torchilin V, Patri AK, Hrkach J, Stern S, et al. (2012) Best practices in cancer nanotechnology: perspective from $\mathrm{NCl}$ nanotechnology alliance. Clin Cancer Res 18: 3229-3241.

3. Aliabadi HM, Elhasi S, Mahmud A, Gulamhusein R, Mahdipoor P, et al. (2007) Encapsulation of hydrophobic drugs in polymeric micelles through co-solvent evaporation: the effect of solvent composition on micellar properties and drug loading. Int J Pharm 329: 158-165.

4. Han $Y$, Tian $H$, He $P$, Chen $X$, Jing $X(2009)$ Insulin nanoparticle preparation and encapsulation into poly(lactic-co-glycolic acid) microspheres by using an anhydrous system. Int J Pharm 378: 159-166.

5. Aliabadi HM, Mahmud A, Sharifabadi AD, Lavasanifar A (2005) Micelles of methoxy poly(ethylene oxide)-b-poly(epsilon-caprolactone) as vehicles for the solubilization and controlled delivery of cyclosporine A. J Control Release 104: 301-311.

6. Gillies ER, Fréchet JM (2003) A new approach towards acid sensitive copolymer micelles for drug delivery. Chem Commun (Camb), pp: 1640-1641.

7. Min Y, Caster JM, Eblan MJ, Wang AZ (2015) Clinical Translation of Nanomedicine. Chem Rev 115: 11147-11190.

8. Sethuraman VA, Bae YH (2007) TAT peptide-based micelle system for potentia active targeting of anti-cancer agents to acidic solid tumors. J Control Release 118: 216-224.

9. Immordino ML, Brusa P, Arpicco S, Stella B, Dosio F, et al. (2003) Preparation, characterization, cytotoxicity and pharmacokinetics of liposomes containing docetaxel. J Control Release 91: 417-429.

10. Gaucher G, Dufresne MH, Sant VP, Kang N, Maysinger D, et al. (2005) Block copolymer micelles: preparation, characterization and application in drug delivery. J Control Release 109: 169-188.

11. Zhang Z, Feng SS (2006) The drug encapsulation efficiency, in vitro drug release, cellular uptake and cytotoxicity of paclitaxel-loaded poly(lactide)tocopheryl polyethylene glycol succinate nanoparticles. Biomaterials 27: 4025 4033.

12. Ventura CA, Tommasini S, Falcone A, Giannone I, Paolino D, et al. (2006) Influence of modified cyclodextrins on solubility and percutaneous absorption of celecoxib through human skin. Int J Pharm 314: 37-45.

13. Nii T, Ishii F (2005) Encapsulation efficiency of water-soluble and insoluble drugs in liposomes prepared by the microencapsulation vesicle method. Int $J$ Pharm 298: 198-205. 